\title{
P-0814 - Factors associated with subsequent glycemic control in patients who discontinued diabetes care
}

Noriko Ihana-Sugiyama1, Takehiro Sugiyama1,2,3,4, Kenjiro Imai' ${ }^{1}$, Ayako Yanagisawa1,2,

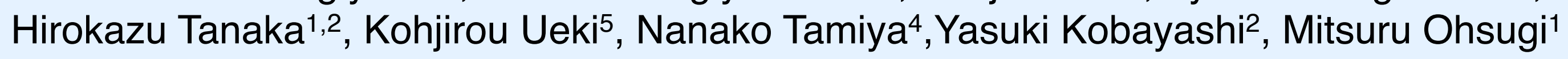

1 Diabetes and Metabolism Information Center, Research Institute, National Center for Global Health and Medicine

2 Department of Public Health/Health Policy, Graduate School of Medicine, the University of Tokyo

3 Health Services Research and Development Center, University of Tsukuba

4 Department of Health Services Research, Faculty of Medicine, University of Tsukuba

Introduction

5 Diabetes Research Center, Research Institute, National Center for Global Health and Medicine

Discontinuation of diabetes care devastates glycemic control and causes complications; however, few studies have investigated the effects of discontinuation on subsequent glycemic control and its associated factors. The aim of this study was to estimate the effects of discontinuation on subsequent glycemic control and to investigate its associated factors.

\section{Methods}

This retrospective cohort study included data from health insurance claims and health checkups provided by employer-sponsored health insurers in Japan via the JMDC Co., Ltd between January 2005 and April 2018.

Patients with diabetes were identified according to antidiabetic medication prescription at least once during the follow-up or a glycohemoglobin (HbA1c) level of $\geq 6.5 \%$ at baseline health checkups.

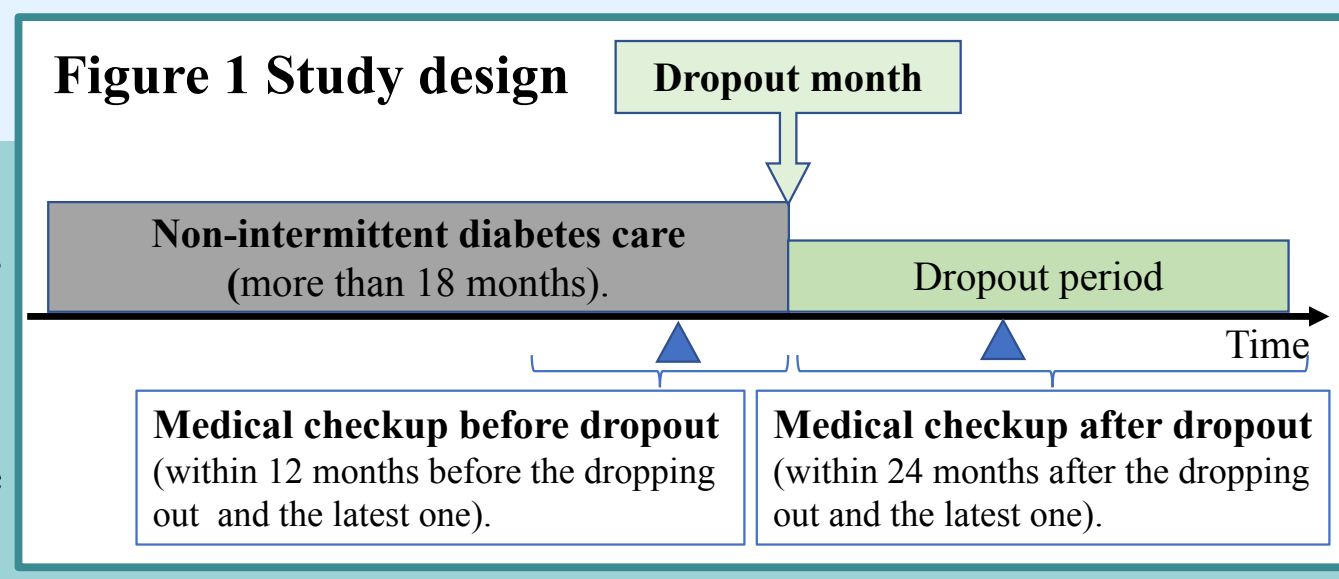

Patients who continued diabetes care (with $\mathrm{HbAl}$ c/glycoalbumin measurements and/or antidiabetic medication at least once in 3 months) for $\geq 18$ months and subsequently dropout of diabetes care (intermission or cessation of treatment for $>3$ months) were included, considering that most frequent medical care intervals for outpatient visits were $\leq 3$ months in Japan. We included patients with HbA1c levels and BMI obtained from the health checkup data at both $\leq 12$ months before and $\leq 24$ months after the month (Fig. 1).

Patients were divided into three groups according to the baseline HbAlc levels: Group 1, $<7.0 \%$; Group 2, $\geq 7.0 \%-8.0 \%$; and Group 3: $\geq 8.0 \%$.

A one-way analysis of variance was used to evaluate changes in HbAlc levels. Multiple logistic regression analysis was used to investigate factors related to higher $\mathrm{HbA} 1 \mathrm{c}$ levels after the dropout (HbAlc of $\geq 8 \%$ ) and baseline characteristics.

\section{$\underline{\text { Results }}$}

Among 141,010 patients, $16.5 \%$ discontinued diabetic treatment during the observation. 2,161 of them had information from medical checkups. $56.0 \%$ received antidiabetic medication at the dropout month (Fig. 2). The mean (standard deviation) age, baseline $\mathrm{HbA1c}$, and baseline BMI was 52.1 (8.0) years, $6.8(1.2 \%)$, and 26.2 (4.5), respectively. During the dropout period, HbAlc levels and BMI were $7.4 \%(1.9 \%)$ and 26.0 (4.5), respectively (Table 1). Changes in HbAlc levels were significantly different among the groups (Group 1, 0.47\%; Group 2, $0.87 \%$; and Group 3, 0.66\%; P < 0.001; Fig. 2A). Follow-up HbA1c levels were also different among the groups (Fig. 3B).

Higher baseline HbAlc levels and a BMI of $>25$, a longer interval between the dropout month and the follow-up health checkup, and antidiabetic medication use, particularly sulfonylurea and insulin, were associated with poor glycemic control after dropping out (Table 2).

\section{Table 1. Characteristics of patients included in this study}

\section{Age, year}

Sex, male

$\mathbf{N}=\mathbf{2 , 1 6 1}$

Type of diabetes

Type 1

Type 2 / others

Baseline HbA1c, \%

Baseline BMI

Non-intermittent of diabetes care before the dropout, month

$52.1(8.0)$

$1,878(86.9)$

$11(5.0)$

$6.8(1.2)$

$26.2(4.5)$

$32.9(17.0)$

Antidiabetic medication

No antidiabetic medication

Any antidiabetic medication

Sulfonylurea

Insulin

Both sulfonylurea and insulin

$951(44.0)$

$1,210(56.0)$

$283(13.1)$

$114(5.3)$

$10(0.5)$
$2,150(95.0)$

Total number of patients who continued diabetic treatment more than 18 months during the observation period. $\mathrm{N}=141,010$

Patients who discontinued diabetic treatment $>3$ months during the observation period. $\mathrm{N}=57,532$

\begin{tabular}{|l|l|}
\hline & $\begin{array}{l}\text { Patients who ended observation } \\
3 \text { months after discontinuing dia }\end{array}$ \\
- Patients who received antidiabetic drugs at the dropout month. \\
Patients who had medical checkup information before an
\end{tabular}

Fig. 2

Flow chart of patients selection.

Table 2. Multivariable analysis investigating factors associated with poor glycemic control (HbA1c $\geq 8 \%$ ) during the dropout period.
Baseline HbA1c
Baseline BMI $>25$
3.83
1.37
1.03
$3.26-4.49$
$1.03-1.83$
$0.67-1.83$
Sex
Interval from the dropout month
to follow-up health examination
1.08
$1.05-1.10$
$<0.001$
0.029
0.896
Antidiabetic medication
No medication
Antidiabetic drug excluding
sulfonylurea and insulin
fonylurea
Insulin

Odds ratio

95\% Conf. Interval

P value

Odds ratios adjusted for age, sex, baseline $\mathrm{HbAlC}$, baseline BMI, interval between baseline hea

dropout interval between dropping out and follow-up health examination, and antidiabetic drugs.
Antidiabetic drug excluding sulfonylurea and insulin

Biganide

Dipeptidyl peptidase-4 inhibitors

Sodium glucose cotransporter 2 inhibitors

Alpha glucosidase inhibitors

Thiazolizine

Glucagon-like peptide-1 receptor agonist

HbA1c during dropout, $\%$

BMI during dropout

Interval from the dropout month to follow-up health examination, month

$878(38.4)$

$518(24.0)$

$715(33.1)$

$120(5.6)$

$189(8.8)$

$193(8.9)$

$22(1.0)$

$7.4(1.9)$

$26.0(4.5)$

$7.6(6.5)$

\section{Figure 3 Change and follow-up HbA1c} during the dropout period

A. Change HbA1c (\%) B. Follow-up HbA1c (\%) $\mathrm{P}<0.001$ $\mathrm{P}<0.001$ This study was supported by JSPS KAKENHI Grant Number 19K19451. 